\title{
Evaluation de l'efficacité d'un traitement spécifique contre les diarrhées du chamelon
}

\author{
M. Bengoumi ${ }^{1}$ J. Berrada ${ }^{1}$ F. de La Farge ${ }^{2}$ \\ K. Hidane ${ }^{3}$ B. Faye ${ }^{4}$
}

Mot-clés

D romadaire - Jeune animal - Diarrhée Thérapeutique - Réhydratation.

\begin{abstract}
Résumé
L'efficacité d'une formule d'un réhydratant associée à un traitement anti-infectieux a été évaluée chez 21 chamelons diarrhéiques dans la région de Dakhla et de Guelmim au Sud du Maroc. Un examen clinique et des prélèvements de sang et de matières fécales ont été réalisés avant et après trois jours de traitement. Les résultats ont montré que ce traitement a été efficace contre les diarrhées du chamelon. Les paramètres cliniques ont été rétablis et les signes cliniques et biochimiques de la déshydratation ont été corrigés. En effet, le traitement de trois jours a entraîné une amélioration de l'état général avec diminution de la température corporelle, reprise de l'appétit, une bonne réactivité et disparition de l'énophtalmie et de la persistance du pli cutané. Pour les selles, des effets significatifs ont été notés sur la consistance, la couleur, I'odeur et la présence de substances étrangères. Pour les paramètres sanguins, le traitement a induit une baisse significative de l'hématocrite, une augmentation de la natrémie, de la magnésémie, de la bicarbonatémie et de la glycémie.
\end{abstract}

\section{INTRO DUCTION}

La mortalité des chamelons est l'une des contraintes majeures au développement de l'élevage camelin. Elle peut atteindre plus de 50 p. 100 dans certains pays avec une moyenne de 20 à 30 p. 100. Les diarrhées sont les principales causes de morbidité et de mortalité des chamelons avant sevrage ; elles entrainent des pertes économiques sévères. L'étude de la physiopathologie des diarrhées montre qu'elles entraînent une déshydratation hypotonique (hémoconcentration, hyponatrémie, légère hyperkaliémie) et une acidose compensée (2). A la lumière de ces résultats, la formule d'un traitement spécifique associant un réhydratant et des anti-infectieux a été élaborée. L'objectif du présent travail a été d'évaluer l'efficacité de ce traitement chez des chamelons diarrhéiques.

\section{MATERIEL ET METHODES}

\section{Matériel}

L'étude a été réalisée dans la province de Dakhla et en particulier dans les régions d'Aousserd et de Guelmim. Le choix de la région de Dakhla a été imposé par la présence de la majorité des troupeaux du Sahara dans les parcours riches de la région d'Aousserd. Ce travail a été réalisé entre janvier et avril 1999. Le choix de cette

1. Institut agronomique et vétérinaire Hassan II, BP 6202, Rabat, Maroc

2. Direction provinciale de l'agriculture, Dakhla, Maroc

3. CHU, Toulouse-Rangueil, 31054 Toulouse, France

4. Cirad-emvt, Productions animales, TA30/A, 34398 Montpellier Cedex 5, France période s'explique par la saison des chamelages qui s'étend de décembre à mars. Cette étude a concerné 21 chamelons âgés de 15 à 90 jours avec un poids de 30 à $75 \mathrm{~kg}$. Le choix des troupeaux a été dicté par la présence de chamelons diarrhéiques. Ces derniers ont été soumis à un examen clinique complet et à des prélèvements de sang et de fèces avant le traitement et un jour après la fin de celui-ci.

Comme traitement, les chamelons diarrhéiques ont reçu un sachet de réhydratant par jour pendant trois jours. Le sachet était dilué dans 2,5 1 d'eau et était réparti en quatre prises. Pendant la période du traitement, le lait a été supprimé.

\section{Méthodes}

Dès le prélèvement, l'hématocrite a été déterminé après centrifugation du sang total pendant 15 min à $3000 \mathrm{~g}$. Les paramètres biochimiques témoins des perturbations métaboliques ont été analysés sur un auto-analyseur Ektachem Kodak XR 700. L'analyse bactériologique des matières fécales s'est focalisée sur la recherche de salmonelles et de colibacilles selon les méthodes classiques. Le traitement statistique s'est appuyé sur des méthodes d'analyse de la variance à un critère de classification (Anova).

\section{RESU LTATS ET DISCUSSION}

Les résultats concernant l'examen clinique des animaux avant et après traitement sont présentés dans le tableau I. Les diarrhées ont affecté l'état général des animaux entraînant de l'hyperthermie, une diminution de l'appétit, un réflexe de succion faible ou désorganisé, un état d'abattement et de prostration et parfois même de décubitus. Les signes de déshydratation ont été bien marqués, en particulier la persistance du pli cutané et l'enfoncement du globe oculaire ou 
énophtalmie. Après trois jours de traitement, une nette amélioration de l'état général a été notée, en particulier la disparition de l'hyperthermie et de l'abattement et la reprise de l'appétit avec un bon réflexe de succion. Les signes de déshydratation, c'est-à-dire persistance du pli cutané, froideur de la cavité buccale et énophtalmie, ont été corrigés. Ces effets auraient été liés à l'utilisation d'un traitement anti-infectieux et surtout à la restauration de l'équilibre hydroélectrolytique et à l'apport de glucose comme source d'énergie.

Les caractéristiques des selles sont restées de loin les symptômes les plus évidents et les plus pathognomoniques (tableau II). En effet, les selles normales d'un chamelon ont une consistance pâteuse, une couleur verdâtre, une odeur légèrement lactique avec absence de substances étrangères telles que le mucus, la fibrine ou le sang (2). Les diarrhées ont provoqué une modification très importante des selles avec une consistance liquide ou en bouillie, une couleur jaune verdâtre à jaunâtre, une odeur putride et la présence de mucus ou de sang. Ces perturbations sont comparables à celles observées chez le veau (8). Les pertes hydriques par voie fécale et les fermentations intestinales associées à l'entérite ont été à l'origine de ces modifications, plus marquées chez les jeunes en allaitement total, en raison de la putréfaction rapide des protéines du lait. En effet, les toxines produites par les agents infectieux induisent une hypersécrétion intestinale et une fuite d'eau des électrolytes vers la lumière intestinale. Il en résulte une augmentation de la fluidité des fèces et de la fréquence de leur émission souvent accompagnée d'une hypermotricité intestinale (8).

Le traitement a entraîné une modification significative des selles qui sont devenues normales (voir plus haut). L'effet du traitement

Tableau I

Effet du traitement sur certains paramètres cliniques

\begin{tabular}{|c|c|c|c|c|c|}
\hline \multirow[t]{2}{*}{ Paramètres } & \multirow[t]{2}{*}{ Etat } & \multicolumn{2}{|c|}{ Avant le traitement } & \multicolumn{2}{|c|}{ Après le traitement } \\
\hline & & Effectif & $\%$ & Effectif & $\%$ \\
\hline Appétit & $\begin{array}{l}\text { Bon } \\
\text { Moyen } \\
\text { Mauvais }\end{array}$ & $\begin{array}{r}1 \\
15 \\
5\end{array}$ & $\begin{array}{r}5 \\
71 \\
24\end{array}$ & $\begin{array}{r}18 \\
3 \\
0\end{array}$ & $\begin{array}{r}86 \\
14 \\
0\end{array}$ \\
\hline Attitude & $\begin{array}{l}\text { Eveillé } \\
\text { Abattu } \\
\text { Décubitus }\end{array}$ & $\begin{array}{r}8 \\
11 \\
2\end{array}$ & $\begin{array}{l}38 \\
52 \\
10\end{array}$ & $\begin{array}{r}19 \\
2 \\
0\end{array}$ & $\begin{array}{r}90 \\
10 \\
0\end{array}$ \\
\hline Pli cutané & $\begin{array}{l}<5 \mathrm{~s} \\
5-10 \mathrm{~s} \\
\text { Persistant }\end{array}$ & $\begin{array}{r}0 \\
12 \\
9\end{array}$ & $\begin{array}{r}0 \\
57 \\
43\end{array}$ & $\begin{array}{r}19 \\
2 \\
0\end{array}$ & $\begin{array}{r}90 \\
10 \\
0\end{array}$ \\
\hline Enophtalmie & $\begin{array}{l}\text { Absence } \\
\text { Légère } \\
\text { Sévère }\end{array}$ & $\begin{array}{r}2 \\
17 \\
2\end{array}$ & $\begin{array}{l}10 \\
81 \\
10\end{array}$ & $\begin{array}{r}19 \\
2 \\
0\end{array}$ & $\begin{array}{r}90 \\
10 \\
0\end{array}$ \\
\hline Réflexe de succion & $\begin{array}{l}\text { Puissant } \\
\text { Faible } \\
\text { Désorganisé }\end{array}$ & $\begin{array}{r}5 \\
14 \\
2\end{array}$ & $\begin{array}{l}24 \\
67 \\
10\end{array}$ & $\begin{array}{r}16 \\
5 \\
0\end{array}$ & $\begin{array}{r}76 \\
24 \\
0\end{array}$ \\
\hline Température & $\begin{array}{l}<38 \\
38-39 \\
>39\end{array}$ & $\begin{array}{r}3 \\
11 \\
7\end{array}$ & $\begin{array}{l}14 \\
52 \\
33\end{array}$ & $\begin{array}{r}13 \\
8 \\
0\end{array}$ & $\begin{array}{r}62 \\
38 \\
0\end{array}$ \\
\hline
\end{tabular}

\section{Tableau II}

Effet du traitement sur les caractéristiques des selles

\begin{tabular}{|c|c|c|c|c|c|}
\hline \multirow[t]{2}{*}{ Paramètres } & \multirow[t]{2}{*}{ Etat } & \multicolumn{2}{|c|}{ Avant le traitement } & \multicolumn{2}{|c|}{ Après le traitement } \\
\hline & & Effectif & $\%$ & Effectif & $\%$ \\
\hline Consistance & $\begin{array}{l}\text { Pâteuse } \\
\text { En bouillie } \\
\text { Liquide }\end{array}$ & $\begin{array}{r}1 \\
15 \\
5\end{array}$ & $\begin{array}{r}5 \\
71 \\
24\end{array}$ & $\begin{array}{r}18 \\
3 \\
0\end{array}$ & $\begin{array}{r}86 \\
14 \\
0\end{array}$ \\
\hline Couleur & $\begin{array}{l}\text { Verdâtre } \\
\text { Jaune verdâtre } \\
\text { Jaunâtre }\end{array}$ & $\begin{array}{r}3 \\
12 \\
6\end{array}$ & $\begin{array}{l}14 \\
57 \\
29\end{array}$ & $\begin{array}{r}14 \\
7 \\
0\end{array}$ & $\begin{array}{r}67 \\
33 \\
0\end{array}$ \\
\hline O deur & $\begin{array}{l}\text { Normale } \\
\text { Putride }\end{array}$ & $\begin{array}{r}1 \\
20\end{array}$ & $\begin{array}{r}5 \\
95\end{array}$ & $\begin{array}{r}13 \\
8\end{array}$ & $\begin{array}{l}62 \\
38\end{array}$ \\
\hline $\begin{array}{l}\text { Substances } \\
\text { étrangères }\end{array}$ & $\begin{array}{l}\text { Absence } \\
\text { Mucus } \\
\text { Fibrine } \\
\text { Sang }\end{array}$ & $\begin{array}{r}12 \\
7 \\
0 \\
2\end{array}$ & $\begin{array}{r}57 \\
33 \\
0 \\
10\end{array}$ & $\begin{array}{r}17 \\
4 \\
0 \\
0\end{array}$ & $\begin{array}{r}81 \\
19 \\
0 \\
0\end{array}$ \\
\hline
\end{tabular}


sur les caractéristiques des selles s'expliquerait par la lutte contre les bactéries et, par conséquent, leurs toxines par les anti-infectieux utilisés (8).

Chez le chamelon, les infections bactériennes responsables des diarrhées sont essentiellement la salmonellose, la colibacillose et les entérotoxémies. Les études précédentes menées au Sud du Maroc ont permis d'isoler plusieurs sérotypes de salmonelles. Cependant, c'est la première fois qu'Escherichia coli $\mathrm{K} 99^{+}$a été isolée chez le dromadaire au Maroc (tableau III). Ces résultats sont comparables à ceux rapportés dans les autres pays (5).

Les résultats des paramètres sanguins sont présentés dans le tableau IV. Les diarrhées ont eu un effet significatif sur certains paramètres sanguins, notamment sur l'augmentation de l'hématocrite, de l'urémie et de la créatininémie, d'une part et sur la baisse de la natrémie, de la bicarbonatémie, de la glycémie et de la magnésémie, d'autre part.

L'hématocrite renseigne globalement sur le volume des liquides circulants (hémodilution, hémoconcentration) au cours des privations ou des apports hydriques. La fuite d'eau dans les selles se répercute directement dans le liquide extracellulaire, en particulier le plasma. L'hémoconcentration qui s'ensuit se traduit par une

\section{Tableau III}

Principales bactéries isolées avant le traitement

\begin{tabular}{lrr}
\hline Agent infectieux & Effectif $(\mathbf{n}=\mathbf{1 7})$ & \% \\
\hline Escherichia coli K99+ & 5 & 29 \\
Escherichia coli K99- & 11 & 65 \\
Salmonella sp. & 1 & 6 \\
Gram - non entérobactérie & 2 & 12
\end{tabular}

élévation des éléments corpusculaires du sang. L'hématocrite a diminué en moyenne de sept points après trois jours de traitement (33 vs 26). Cette diminution était liée aux apports hydriques qui étaient de 2,5 l par jour, mais aussi à la diminution des pertes dans les matières fécales.

Chez les chamelons malades, la natrémie est légèrement plus basse mais dans une proportion moins importante que celle décrite chez le veau diarrhéique (3). L'hyponatrémie serait beaucoup plus importante, mais elle est masquée par l'hypovolémie (1). L'hyponatrémie est la conséquence directe de la fuite du sodium dans les matières fécales. Il s'agirait donc d'une déshydratation de type hypotonique, caractérisée par la diminution de la pression osmotique. L'association du traitement anti-infectieux et de l'apport du sodium a permis de lutter conte les fuites et de rétablir la natrémie. La kaliémie et la chlorurémie n'ont pas été affectées par les diarrhées ou le traitement, ce qui confirme les observations faites précédemment (2). Chez le veau diarrhéique, l'élévation de la kaliémie est plus importante (7). Cette hyperkaliémie est liée au passage des ions $\mathrm{K}^{+}$du milieu intracellulaire vers le compartiment extracellulaire, contribuant ainsi, par l'échange avec l'ion $\mathrm{H}^{+}$, à compenser l'acidose métabolique. L'influence des diarrhées sur la chlorurémie n'est pas claire et les résultats de la littérature sont contradictoires (7). La baisse de la concentration plasmatique des bicarbonates chez les chamelons diarrhéiques était comparable à celles rapportées chez les veaux (7) et chez les chamelons (2). L'hypobicarbonatémie est liée à la compensation de l'acidose métabolique consécutive à la fuite importante du sodium et des bicarbonates dans les matières fécales et à la production d'acides organiques en particulier l'acide lactique (3). Le traitement a eu un effet significatif sur la concentration plasmatique des bicarbonates qui a augmenté de 18 à $22 \mathrm{mmol} / \mathrm{l}$. Cet effet aurait été lié à la diminution des pertes fécales et à l'apport du sodium, électrolyte alcalinisant par excellence. Par ailleurs, l'apport de glucose permet également de limiter la production d'acides.

\section{Tableau IV}

Effet du traitement sur certains paramètres sanguins (moyenne + écart-type)

\begin{tabular}{|c|c|c|c|}
\hline Paramètres & Avant le traitement & Après le traitement & Anova \\
\hline Hématocrite (I/I) & $33 \pm 2$ & $26 \pm 2$ & $* * *$ \\
\hline Sodium (mmol/l) & $150 \pm 4$ & $156 \pm 5$ & $*$ \\
\hline Potassium (mmol/l) & $5,6 \pm 0,7$ & $5,4 \pm 0,4$ & $n s^{1}$ \\
\hline Chlorures(mmol/l) & $109 \pm 6$ & $109 \pm 8$ & ns \\
\hline Bicarbonates (mmol/l) & $18,3 \pm 3,6$ & $22,2 \pm 2,4$ & $* * *$ \\
\hline Calcium (mmol/l) & $2,50 \pm 0,11$ & $2,57 \pm 0,07$ & ns \\
\hline Phosphates (mmol/l) & $3,26 \pm 0,42$ & $3,35 \pm 0,27$ & ns \\
\hline Magnésium (mmol/l) & $0,84 \pm 0,10$ & $0,97 \pm 0,12$ & $*$ \\
\hline Protéines $(\mathrm{g} / \mathrm{l})$ & $61 \pm 9$ & $56 \pm 7$ & ns \\
\hline U rée $(\mathrm{mmol} / \mathrm{l})$ & $7,8 \pm 7,3$ & $4,6 \pm 1,2$ & ns \\
\hline Créatinine (mmol/l) & $132 \pm 86$ & $98 \pm 28$ & ns \\
\hline Bilirubine (mmol/l) & $3,5 \pm 0,9$ & $2,7 \pm 0,5$ & ns \\
\hline Glucose $(\mathrm{mmol} / \mathrm{l})$ & $6,5 \pm 0,5$ & $7,2 \pm 0,6$ & $* *$ \\
\hline Triglycérides (mmol/l) & $0,82 \pm 0,38$ & $0,83 \pm 0,34$ & ns \\
\hline Cholestérol (mmol/l) & $1,48 \pm 0,95$ & $1,15 \pm 0,41$ & ns \\
\hline Asat (U/I) & $125 \pm 45$ & $124 \pm 29$ & ns \\
\hline Alat (U/I) & $21 \pm 6$ & $20 \pm 4$ & ns \\
\hline Ldh (U/I) & $2461 \pm 534$ & $2367 \pm 476$ & ns \\
\hline Pal (U/I) & $375 \pm 70$ & $393 \pm 62$ & ns \\
\hline Ggt $(\mathrm{U} / \mathrm{l})$ & $16 \pm 3$ & $17 \pm 2$ & ns \\
\hline $\mathrm{Ck}(\mathrm{U} / \mathrm{I})$ & $158 \pm 53$ & $140 \pm 38$ & ns \\
\hline
\end{tabular}

$* \mathrm{P}<0,05 ; * * \mathrm{P}<0,01 ; * * * \mathrm{P}<0,001$

1. $\mathrm{P}>0,05$ 
L'apport de magnésium dans le traitement a induit une légère augmentation de la magnésémie. La fuite du magnésium lors des diarrhées contribuerait à l'abattement des animaux (6).

Les diarrhées n'ont pas semblé affecter la calcémie et la phosphatémie. En effet, ses deux paramètres sont soumis à une régulation hormonale et leur déficit est comblé par une diminution de l'excrétion et surtout une mobilisation des réserves osseuses. Des observations similaires ont été observées chez les veaux (7) et chez les chamelons (2).

La glycémie du dromadaire est plus élevée que celle des autres ruminants ou herbivores. La néoglucogenèse est très active chez le dromadaire. Les valeurs observées dans cette étude chez les animaux sains sont comparables aux résultats déjà publiés pour cette espèce (4).

L'inappétence et la faible absorption du glucose étaient responsables de l'hypoglycémie observée lors des diarrhées. L'apport du glucose et du propionate qui est un stimulateur de la néoglucogenèse explique l'augmentation significative de la glycémie après le traitement. Généralement, les diarrhées se traduisent par une élévation de la protéinémie, de l'urémie et de la créatininémie par un simple effet d'hypovolémie liée à la déshydratation. Celle-ci entraîne une diminution de la diurèse et une accumulation des produits issus du catabolisme protéique dans le sang est alors observée (6). Ces phénomènes apparaissent moins marqués chez le chamelon, comparé au veau, bien que chez les chamelons les plus déshydratés, l'urémie avait atteint $29 \mathrm{mmol} / \mathrm{l}$. La légère diminution non significative de l'urémie après le traitement aurait été liée au rétablissement de l'équilibre hydrique et, par conséquent, de l'excrétion rénale.

Les diarrhées et le traitement n'ont pas semblé influencer la fonction hépatobiliaire comme en témoignait la stabilité de la bilirubinémie. Les activités plasmatiques de l'Asat, l'Alat, la Ggt, la $\mathrm{Ck}$, la Ldh et les Pal n'ont pas été significativement influencées par les diarrhées ou le traitement. Il en découle que les tissus hépatique, musculaire, cardiaque, osseux ou pulmonaire n'ont pas souffert de lésions cellulaires lors des diarrhées ou à la suite du traitement (6).

\section{Summary}

Bengoumi M., Berrada J., De La Farge F., Hidane K., Faye B. Assessment of the efficacy of a specific treatment against camel calf diarrhea

The efficacy of a rehydrating formula combined with an anti-infective treatment was assessed in 21 camel calves in Dakhla and Guelmim regions in South Morocco. Clinical examination as well as blood and feces sampling were performed before and after three days' treatment. Results showed the treatment to be effective against camel calf diarrhea. Clinical parameters were recovered, and clinical and biochemical signs of dehydration were corrected. Actually, overall health improvement with decrease in body temperature, appetite recovery, good reactivity, enophthalmia and skin-fold disappearance were observed following the three-day treatment. Significant effects were noted on feces consistency, color, odor and presence of foreign substance. Similarly, concerning blood parameters, the packed cell volume significantly decreased, whereas blood sodium, magnesium, bicarbonate and glucosis increased.

Key words: Dromedary - Young animal - Diarrhea - Therapy Rehydration.

\section{CONCLUSION}

Ces premiers résultats montrent que l'association d'un anti-infectieux et d'un réhydratant est efficace dans le traitement des diarrhées du chamelon. Les résultats des enquêtes écopathologiques permettront de proposer une stratégie de lutte contre les diarrhées du chamelon basée sur l'utilisation adéquate de ce traitement mis au point pour la première fois chez cette espèce. D'autres études sur les agents pathogènes permettraient d'élaborer des vaccins spécifiques comme chez les autres espèces domestiques.

\section{BIBLIO GRAPHIE}

1. BENGOUMI M., RIAD F., GIRY G., DE LA FARGE F., SAFWATE A., DAVICCO M.-J., BARLET J.-P., 1993. Hormonal control of water and sodium in plasma and urine of camels during dehydration and rehydration. Gen. Comp. Endocrinol., 89: 378-386.

2. BENGOUMI M., BERRADA J., ROCHDI M., HIDANE K., DE LA FARGE F., FAYE B., 1998. Physiopathologie des diarrhées du chamelon au Maroc : Signes cliniques et perturbations métaboliques. Revue Elev. Méd. vét. Pays trop., $51:$ 277-281.

3. DEMIGNE C., REMESY C., 1980. Evidence of different types of acidosis associated with diarrhea in the neonatal calf. Ann. Rech. vét., 11: $267-272$

4. ELIAS E., YAGIL R., 1984. Haematological and serum biochemical values in lactating camels (Camelus dromedarius) and their newborn. Refuah Vet., 41: 7-13.

5. FAYE B., BENGOUMI M., Ed., 1999. Guide de l'élevage du dromadaire (en arabe). Libourne, France, Sanofi.

6. KANEKO J.J., 1989. Clinical biochemistry of domestic animals, $4 \mathrm{~h}$ ed. New York, NY, USA, Academic Press.

7. MAACH L., GRUNDER H.D., BOUJIJA A., 1995. La diarrhée néonatale du veau. Etude physiopathologique. Maghreb Vét., 7 : 30.

8. VALLET A., 1983. Rôle des facteurs du milieu dans la pathologie du veau nouveau-né. Epidémiologie et santé animale. Bull. AEEM A, 3 : 5-24.

\section{Resumen}

Bengoumi M., Berrada J., De La Farge F., Hidane K., Faye B. Evaluación de la eficiencia de un tratamiento específico contra las diarreas del camello joven

Se evaluó la eficiencia de una fórmula de un hidratante asociado a un tratamiento anti infeccioso, en 21 camellos jóvenes diarreicos en la región de Dakhla y de Guelmin, al sur de Marruecos. Se realizó un examen clínico, así como tomas de sangre y de materias fecales, antes y tres días después del tratamiento. Los resultados muestran que este tratamiento es eficaz contra las diarreas en el camello joven. Los parámetros clínicos fueron restablecidos y los signos clínicos y bioquímicas de la deshidratación fueron corregidos. En efecto, el tratamiento de tres días llevó a una mejoría del estado general, con disminución de la temperatura corporal, reaparición del apetito, buena reactividad y desaparición de la endoftalmia y de la persistencia del pliegue cutáneo. En cuanto a las heces, se notaron efectos significativos sobre la consistencia, el color, el olor y la presencia de substancias extrañas. Para los parámetros sanguíneos, el tratamiento llevó a una disminución significativa del hematocrito, un aumento de la natremia, de la magnesemia, del nivel de bicarbonato sanguíneo y de la glicemia.

Palabras clave: Dromedario - Animal joven - Diarrea Terapia - Rehidratación. 